\title{
Evaluation of Investment Alternatives Using Present Value Analysis with Simplified Neutrosophic Sets
}

\author{
Serhat Aydin'1, Cengiz Kahraman², Mehmet Kabak ${ }^{3}$
}

\author{
${ }^{1}$ National Defense University \\ 34149, Istanbul, Turkey \\ E-mail.saydin3@hho.edu.tr \\ ${ }^{2}$ Istanbul Technical University \\ 34367, Istanbul, Turkey \\ E-mail.kahramanc@itu.edu.tr \\ ${ }^{3}$ Gazi University \\ 06570, Ankara, Turkey \\ E-mail.mkabak@gazi.edu.tr
}

cross $^{\text {ref }}$ http://dx.doi.org/10.5755/j01.ee.29.3.19392

\begin{abstract}
Engineering economics is the collection of mathematical techniques that simplify economic comparisons of investment alternatives. Investment analysis is a branch of engineering economics, which focuses on choosing the most profitable investment option for a company. Because of vague, imprecise, and insufficient data in investment analysis, decision makers use fuzzy logic to make evaluation correctly under uncertainty. This paper develops simplified neutrosophic present worth analysis method in order to overcome difficulties in defining the membership functions of investment parameters. A numerical example illustrates the applications of the developed formulas. The results of the developed methodology are compared with classical present value analysis and intuitionistic present value analysis. The comparison results indicate that investment evaluation problem can be tackled by using the proposed methodology effectively.
\end{abstract}

Keywords: Engineering Economics; Investment Analysis; Present Value Analysis; Neutrosophic Sets; Fuzzy Logic.

\section{Introduction}

Engineering economics is the systematic evaluation of the economic merits of proposed solutions to engineering problems. The main objective of engineering economics is to compare the economic value of alternative design options.

Investment analysis problem is a branch of engineering economics which focuses on choosing the most profitable investment option for a company, regarding the allocation of limited resources. Investment analysis methods use some Engineering economics techniques such as present value analysis (PVA), annual value analysis, future value analysis, benefit/cost ratio analysis, rate of return analysis and payback period analysis.

PVA is the most frequently used technique to determine the present value of the money receipts and disbursement. PVA is used to establish the viability of an investment, often using a discount rate that is adjusted according to the fuzzy environment (Carmichael et. al., 2011). If the cash outflows are compared for an investment analysis problem, the alternative with the lowest present value will be chosen. If the cash inflows are compared, the alternative with the highest present value will be chosen. PVA is used frequently because of its ease of calculation and effective results.
Fuzzy sets, developed by Zadeh (1965), have been widely used to incorporate current uncertainty into the mathematical models. Fuzzy sets theory is an extension of the classical set theory. A classical set is defined by a binary function that only accepts the values 0 and 1 meaning that an element fully belongs to a set or does not belong to a set. However, a fuzzy set is defined by a membership function that accepts all the intermediate values 0 and 1 (Nicolas, 2015). Better and detailed definition of membership functions of investment parameters can be provided by the fuzzy set theory.

In recent years, ordinary fuzzy sets have been extended to new types (Kahraman et al., 2016). Zadeh (1975) introduced type-2 fuzzy sets in 1975. Type-2 fuzzy sets incorporate uncertainty of the membership function into the fuzzy set theory. Interval-valued fuzzy sets which are a special case of type- 2 fuzzy set were introduced independently by Zadeh (1975), Jahn (1975), and Sambuc (1975). Intuitionistic fuzzy sets (IFSs), developed by Atanassov (1986), include the membership value as well as the non-membership value. Yager (1986) introduced fuzzy multi-sets theory. The theory represents multiple occurrences of a subject item with degrees of relevance and it has been studied in relation to a variety of information systems including relational database (Ejegwa, 2014). Hesitant fuzzy sets (HFSs), initially developed by 
Torra (2010) are the extensions of ordinary fuzzy sets, which handle the situations where a set of values are possible for the membership of a single element. F. Smarandache (1998) proposed neutrosophic sets (NS) by adding an independent indeterminacy-membership function to intuitionistic fuzzy sets. The concept of neutrosophic set is a generalization of classic set, ordinary fuzzy set, intuitionistic fuzzy set and interval intuitionistic fuzzy set (Broumi \& Smarandache, 2014). Neutrosophic sets introduce a new component called "indeterminacy", and carry more information than other fuzzy sets (Wen \& Cheng, 2013). A neutrosophic set is expressed by three parameters, which are called truthiness, indeterminacy and falsity. Truthiness and falsity correspond to membership and nonmembership in an intuitionistic fuzzy set. Indeterminacy is a new concept informing the degree of decision makers' neutral thoughts about a judgement. Neutrosophic sets are an extension of intuitionistic fuzzy sets which are presently the most used extension of ordinary fuzzy sets in the literature. Neutrosophic point of view to investment decision problems is a new research area. The other extensions of ordinary fuzzy sets such as type-2 fuzzy sets (Demircan,2016), hesitant fuzzy sets (Kahraman et al., 2015), intuitionistic fuzzy sets (Kahraman et al., 2015) and Pythagorean fuzzy sets (Kahraman et al., 2018) have been already used in investment analysis decision modeling. Hence, we decided to apply neutrosophic sets and neutrosophic data to PVA for comparative analyses with other fuzzy PVAs.

In real life problems, there are difficulties in reaching precise and complete data. For instance, future cash flows alternative lives, and interest rates cannot be known precisely in investment analyses. Therefore, there is a need for a fuzzy approach to investment analysis technique to capture these uncertainties. An uncertain environment should be defined by the parameters which are composed of decision makers' beliefs about the truthiness, indeterminacy and falsity. Therefore, we propose a novel neutrosophic fuzzy PVA approach that can capture the uncertainties of investment parameters in this paper.

The rest of this paper is organized as follows. First, a literature review on fuzzy PVA models is given. Then some concepts of simplified neutrosophic sets are given. Next, PVA with simplified neutrosophic sets is presented. A numerical illustration and comparisons with classical PVA and intuitionistic PVA are presented. Finally, the conclusion is given.

\section{Literature Review: Fuzzy PVA}

Fuzzy engineering economics models have been recently proposed by several authors as an alternative to the conventional engineering economics models. In literature, there have been many studies about fuzzy engineering economics models. As we developed PVA model for investment evaluation using neutrosophic sets, we especially performed literature survey about PVA models using different extension types of fuzzy sets.

Buckley (1987) developed the fuzzy future value and PVA, using both fuzzy cash flows and fuzzy interest rates over $n$ periods, where $n$ may be crisp or fuzzy. Ward (1985) developed fuzzy PVA by using trapezoidal fuzzy cash flows. Kaufmann and Gupta (1988) proposed a fuzzy present value method for investment alternative selection. Chiu and Park (1994) stated cash flow analysis and interest rates analysis representing triangular fuzzy numbers and developed fuzzy PVA. Chan et al. (2000) proposed evaluation methodologies for technology selection by using economics criterion for fuzzy cash flow analysis. Karsak and Tolga (2001) suggested a fuzzy present value model for financial evaluation of advanced manufacturing system investments. Kahraman et al. (2002) developed the formulas for geometric and trigonometric cash flows with of fuzzy present value; fuzzy equivalent uniform annual value, fuzzy future value, fuzzy benefit-cost ratio, and fuzzy payback period.

Kuchta (2001) considered net present value as a quadratic 0-1 programming problem under fuzziness for R\&D project selection. Sheen (2005) improved formulas for fuzzy present value, fuzzy payback period, fuzzy benefit-cost ratio by using Mellin transformation. Omitaomu and Badiru (2007) evaluated information systems with fuzzy present value analysis based on fuzzy triangular numbers. Kuchta (2008) presented the fuzzy net present value maximization as an objective in project selection problems. Dimitrovski and Matos (2008) proposed the fuzzy present worth analysis for cash flows. Kahraman (2008) edited a book on fuzzy engineering economics methods.

$\mathrm{Xu}$ et al. (2009) proposed a three-objective fuzzy chance-constrained programming model based on fuzzy present value analysis for multi-project and multi-item investment combination. Bhattacharyya et al. (2011) proposed fuzzy multi-objective programming method using fuzzy present value analysis for selection research and development projects. Xu et al. (2012) developed multi-objective decision-making methodology based on fuzzy present value analysis. Kahraman et al. (2015) developed fuzzy PVA formulas based on intuitionistic and HFSs.

To the best of our knowledge, the techniques of engineering economics have not yet been handled by using neutrosophic sets. In this paper, we develop the PVA formulas using simplified neutrosophic sets. Thus, the difficulties in defining the membership functions of investment parameters are substantially reduced. The originality of this paper is handling PVA formulas with neutrosophic sets for the first time.

\section{Preliminaries for Simplified Neutrosophic Sets}

Since the introduction of fuzzy logic, many systems have been developed in order to deal with approximate and uncertain reasoning. Among the latest and most general proposals, the neutrosophic logic, introduced by Smarandache (1998) is a generalization of fuzzy logic and several related systems (Kharal, 2014).

Neutrosophic logic is based on neutrosophy. Fuzzy logic extends classical logic by assigning a membership between 0 and 1 to variables. As a generalization of fuzzy logic, neutrosophic logic introduces a new component called "indeterminacy", and carries more information than fuzzy logic. Each proposition is estimated to have a percentage of truth in subset $T$, a percentage of 
indeterminacy in subset $I$, and a percentage of falsity in subset $F$, where $T, I, F$ are subsets of real numbers in[0,1]. Generally, a neutrosophic set is denoted as $\langle T, I, F\rangle$. An element $\mathrm{x}(t, i, f)$ belongs to the set in the following way: $t$ :truthness, $i$ :indeterminacy, and $f$ : falsity in the set, where $t, i$, and $f$ are real numbers taken from sets $T, I$, and $F$ with no restriction on $T, I, F$, and on their sum $m=t+i+f$.

From scientific or engineering point of view, the neutrosophic set and set-theoretic operators will be difficult to apply in the real application without specific description. Therefore, simplified neutrosophic sets (SNS) are proposed $(\mathrm{Xu}, 2012)$ which is an extension of neutrosophic sets.

Some concepts and definitions of SNS are introduced in the following definitions (Ye, 2014):

Definition 1. Let $X$ be a space of objects, with a generic element in $X$ denoted by $x$. A neutrosophic set $A$ in $X$ is characterized by a truth-membership function $T_{A}(x)$, an indeterminacy membership function $I_{A}(x)$ and a falsity-membership function $F_{A}(x)$. The functions $T_{A}(x), I_{A}(x)$ and $F_{A}(x)$ are real standard or nonstandard subsets of $] 0^{-}, 1^{+}[, \quad$ that is $\left.T_{A}(x): X \rightarrow\right] 0^{-}, 1^{+}\left[, I_{A}(x): X \rightarrow\right] 0^{-}, 1^{+}\left[, F_{A}(x): X \rightarrow\right] 0^{-}, 1^{+}[$ . There is no restriction on the sum of $T_{A}(x), I_{A}(x)$ and $F_{A}(x), \operatorname{so} 0^{-} \leq \sup T_{A}(x)+\sup I_{A}(x)+\sup F_{A}(x) \leq 3^{+}$

Definition 2. If the functions $T_{A}(x), I_{A}(x)$ and $F_{A}(x)$ are singleton subintervals/ subsets in the real standart $\quad[0,1], \quad$ that $\quad$ is $T_{A}(x): X \rightarrow[0,1], I_{A}(x): X \rightarrow[0,1], F_{A}(x): X \rightarrow[0,1]$ Then, a simplification of neutrosophic set $A$ is denoted by $A=\left\{\left\langle x, T_{A}(x), I_{A}(x), F_{A}(x)\right\rangle \mid x \in X\right\}$ which is called a SNS. It is subclass of neutrosophic sets.

For each point $x$ in $X$, we have $T_{A}(x), I_{A}(x), F_{A}(x) \in[0,1]$, and $0 \leq T_{A}(x), I_{A}(x), F_{A}(x) \leq 3$.

$A$ SNS $A=\left\{\left\langle x, T_{A}(x), I_{A}(x), F_{A}(x)\right\rangle \mid x \in X\right\}$ is denoted by simplified symbol $A=\left\langle T_{A}(x), I_{A}(x), F_{A}(x)\right\rangle$.

Definition 3. The SNS $A$ is contained in the other SNS $B, \quad A \subseteq B$ if and only if $T_{A}(x) \leq T_{B}(x), I_{A}(x) \geq I_{B}(x)$, and $F_{A}(x) \geq F_{B}(x)$ for every $x$ in $X$.

Definition 4. Let $A, B$ are two SNSs. Operational relations are defined by,

$$
\begin{aligned}
& A+B=\left\langle T_{A}(x)+T_{B}(x)-T_{A}(x) T_{B}(x), I_{A}(x)+I_{B}(x)-\right. \\
& \left.I_{A}(x) I_{B}(x), F_{A}(x)+F_{B}(x)-F_{A}(x) F_{B}(x)\right\rangle \\
& A . B=\left\langle T_{A}(x) T_{b}(x), I_{A}(x) I_{B}(x), F_{A}(x) F_{B}(x)\right\rangle \\
& \left.\lambda A=\left\langle 1-\left(1-T_{A}(x)\right)^{\lambda}, 1-\left(1-I_{A}(x)\right)^{\lambda}, 1-\left(1-F_{A}(x)\right)^{\lambda}\right\rangle, \lambda\right\rangle 0 \\
& \left.A^{\lambda}=\left\langle T_{A}^{\lambda}(x), I_{A}^{\lambda}(x), F_{A}^{\lambda}(x)\right\rangle, \lambda\right\rangle 0
\end{aligned}
$$

Definition 5. For a SNS $A_{j}(j=1,2, \ldots \ldots, n)$, the simplified neutrosophic weighted arithmetic average aggregation operator is defined by

$$
\begin{aligned}
& F_{w}\left(A_{1}, A_{2}, \ldots, A_{n}\right)=\left\langle 1-\prod_{j=1}^{n}\left(1-T_{A_{j}}(x)\right)^{w_{j}}, 1-\prod_{j=1}^{n}\left(1-I_{A_{j}}(x)\right)^{w_{j}},\right. \\
& \left.1-\prod_{j=1}^{n}\left(1-F_{A_{j}}(x)\right)^{w_{j}}\right\rangle
\end{aligned}
$$

Where $W=\left(w_{1}, w_{2}, \ldots ., w_{n}\right)$ is the weight vector of $A_{j}(j=1,2, \ldots, n), w_{j} \in[0,1]$ and $\sum_{j=1}^{n} W_{j}=1$

Especially, assume $W=(1 / n, 1 / n, \ldots ., 1 / n)$, then $F_{w}$ is called as an arithmetic average operator SNS.

Definition 6. For a SNS $A_{j}(j=1,2, \ldots \ldots, n)$, the simplified neutrosophic weighted geometric average aggregation operator is defined by

$$
\left.G_{w}\left(A_{1}, A_{2}, \ldots, A_{n}\right)=\left\langle\prod_{j=1}^{n} T_{A_{j}}^{w_{j}}(x), \prod_{j=1}^{n} I_{A_{j}}^{w_{j}}(x),\right) \prod_{j=1}^{n} F_{A_{j}}^{w_{j}}(x)\right\rangle
$$

where $W=\left(w_{1}, w_{2}, \ldots . ., w_{n}\right)$ is the weight vector of $A_{j}(j=1,2, \ldots . ., n), w_{j} \in[0,1]$ and $\sum_{j=1}^{n} w_{j}=1$

Especially, assume $\quad W=(1 / n, 1 / n, \ldots ., 1 / n)$, then $G_{w}$ is called as an arithmetic average operator SNSs.

As it is noticed in the above definitions, there is no special symbol to indicate that a set is neutrosophic. In the fuzzy set theory, a tilde is used to indicate a fuzzy set. Hence, to fill this need we use the symbol : $A$ for a neutrosophic set.

\section{Present Value Analysis with Simplified Neutrosophic Sets}

The simplified neutrosophic present worth $(S N P V)$ is calculated by Equation 7 or Equation 8.

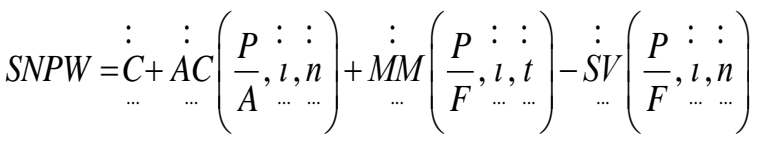

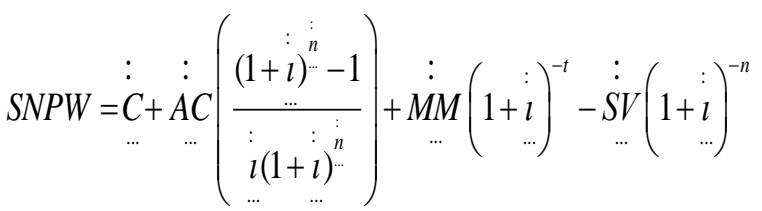

In these formulas, investment parameters will be handled by simplified neutrosophic sets. The handled parameters are Cost $(C)$, Annual Cost $(A C)$, Major Maintenance $(M M)$, Salvage Cost $(S V)$, Interest Rate $(l)$, Useful Life $(n)$, Maintenance time $(t)$. These parameters are expressed by simplified neutrosophic sets except $t$, as follows;

Assuming that $m$ evaluations for each parameter are made: 


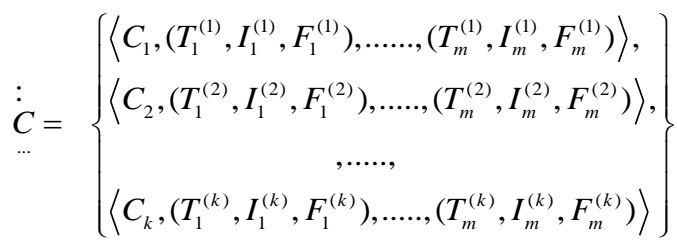

$$
\begin{aligned}
& \begin{array}{l}
: \\
l \\
\cdots
\end{array} \quad=\mathrm{U}_{j=1}^{k} F_{w}\left(\left\langle\begin{array}{l}
: \\
l \\
\ldots j \\
\ldots \ldots \ldots,\left(T_{m}, I_{m}, I_{m}\right)
\end{array}\right)\right) \\
& \begin{array}{l}
n \\
\cdots
\end{array}=\mathrm{U}_{j=1}^{k} F_{w}\left(\left\langle\begin{array}{l}
: \\
n, \ldots,\left(T_{1}, I_{1}, F_{1}\right), \\
\ldots \ldots \ldots,\left(T_{m}, I_{m}, F_{m}\right)
\end{array}\right\rangle\right)
\end{aligned}
$$$$
\dot{S} V=\left\{\begin{array}{c}
\left\langle S V_{1},\left(T_{1}^{(1)}, I_{1}^{(1)}, F_{1}^{(1)}\right), \ldots \ldots,\left(T_{m}^{(1)}, I_{m}^{(1)}, F_{m}^{(1)}\right)\right\rangle, \\
\left\langle S V_{2},\left(T_{1}^{(2)}, I_{1}^{(2)}, F_{1}^{(2)}\right), \ldots . .,\left(T_{m}^{(2)}, I_{m}^{(2)}, F_{m}^{(2)}\right)\right\rangle, \\
, \ldots \ldots, \\
\left\langle S V_{k},\left(T_{1}^{(k)}, I_{1}^{(k)}, F_{1}^{(k)}\right), \ldots . .,\left(T_{m}^{(k)}, I_{m}^{(k)}, F_{m}^{(k)}\right)\right\rangle
\end{array}\right\}
$$$$
\dot{A C}=\left\{\begin{array}{c}
\left\langle A C_{1},\left(T_{1}^{(1)}, I_{1}^{(1)}, F_{1}^{(1)}\right), \ldots \ldots,\left(T_{m}^{(1)}, I_{m}^{(1)}, F_{m}^{(1)}\right)\right\rangle, \\
\left\langle A C_{2},\left(T_{1}^{(2)}, I_{1}^{(2)}, F_{1}^{(2)}\right), \ldots . .,\left(T_{m}^{(2)}, I_{m}^{(2)}, F_{m}^{(2)}\right)\right\rangle, \\
, \ldots . ., \\
\left\langle A C_{k},\left(T_{1}^{(k)}, I_{1}^{(k)}, F_{1}^{(k)}\right), \ldots \ldots,\left(T_{m}^{(k)}, I_{m}^{(k)}, F_{m}^{(k)}\right)\right\rangle
\end{array}\right\}
$$$$
\underset{\cdots}{M} \dot{\ldots}_{\ldots}=\left\{\begin{array}{c}
\left\langle M M_{1},\left(T_{1}^{(1)}, I_{1}^{(1)}, F_{1}^{(1)}\right), \ldots \ldots,\left(T_{m}^{(1)}, I_{m}^{(1)}, F_{m}^{(1)}\right)\right\rangle, \\
\left\langle M M_{2},\left(T_{1}^{(2)}, I_{1}^{(2)}, F_{1}^{(2)}\right), \ldots . .,\left(T_{m}^{(2)}, I_{m}^{(2)}, F_{m}^{(2)}\right)\right\rangle, \\
, \ldots . ., \\
\left\langle M M_{k},\left(T_{1}^{(k)}, I_{1}^{(k)}, F_{1}^{(k)}\right), \ldots . .,\left(T_{m}^{(k)}, I_{m}^{(k)}, F_{m}^{(k)}\right)\right\rangle
\end{array}\right\}
$$$$
: \quad \ldots=\left\{\begin{array}{c}
\left\langle l_{1},\left(T_{1}^{(1)}, I_{1}^{(1)}, F_{1}^{(1)}\right), \ldots \ldots,\left(T_{m}^{(1)}, I_{m}^{(1)}, F_{m}^{(1)}\right)\right\rangle, \\
\left\langle l_{2},\left(T_{1}^{(2)}, I_{1}^{(2)}, F_{1}^{(2)}\right), \ldots . .,\left(T_{m}^{(2)}, I_{m}^{(2)}, F_{m}^{(2)}\right)\right\rangle, \\
, \ldots . ., \\
\left\langle\iota_{k},\left(T_{1}^{(k)}, I_{1}^{(k)}, F_{1}^{(k)}\right), \ldots \ldots,\left(T_{m}^{(k)}, I_{m}^{(k)}, F_{m}^{(k)}\right)\right\rangle
\end{array}\right\}
$$

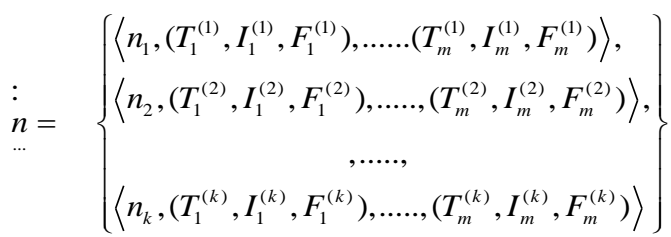

Using the simplified neutrosophic parameters above, the SNPV of an investment alternative can be calculated as follows; Where,

$$
\begin{aligned}
& \left.\begin{array}{l}
\dot{C}=\mathrm{U}_{j=1}^{k} F_{w}\left(\left\langle\begin{array}{l}
\vdots \\
C \\
\ldots j \\
\ldots \ldots \ldots,\left(T_{m}, I_{m}, F_{m}\right)
\end{array}\right)\right.
\end{array}\right)
\end{aligned}
$$

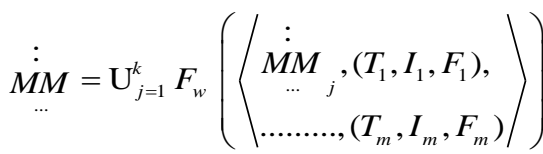

$$
\begin{aligned}
& \stackrel{:}{A C}=\mathrm{U}_{j=1}^{k} F_{w}\left(\left\langle\begin{array}{c}
\vdots \\
A C, \\
\cdots \ldots \ldots,\left(T_{1}, I_{1}, F_{1}\right), \\
\ldots \ldots \ldots .,\left(T_{m}, I_{m}, F_{m}\right)
\end{array}\right)\right) \\
& \stackrel{:}{S V}=\mathrm{U}_{j=1}^{k} F_{w}\left(\left\langle\begin{array}{c}
\vdots \\
S V \\
\ldots, j \\
\ldots \ldots \ldots,\left(T_{m}, I_{m}, F_{m}\right)
\end{array}\right)\right)
\end{aligned}
$$

And then, a deneutrosophication operator is needed to calculating all possible truth-membership values, an indeterminacy membership values and a falsitymembership values.

$$
\begin{aligned}
\stackrel{:}{C}= & \mathrm{U}_{j=1}^{k}\left(\left\langle\begin{array}{l}
C_{j},\left(T_{1}, I_{1}, F_{1}\right), \\
\ldots \ldots \ldots,\left(T_{m}, I_{m}, F_{m}\right)
\end{array}\right)\right)=\mu_{C_{j}} \\
& =\left\langle\mu_{C_{j T}}, \mu_{C_{j l}}, \mu_{C_{j F}}\right\rangle, j=1, \ldots ., k
\end{aligned}
$$

Deneutrosophication value of $\left\langle\mu_{C_{j T}}, \mu_{C_{j l}}, \mu_{C_{j F}}\right\rangle$ is $\operatorname{Def}\left(\mu_{C_{j}}\right)$ which is calculated by Equation 22 developed by us.

$$
D_{(X)}=\frac{T+(1-F)}{2(I+1)}
$$

Now we have possible values and deneutrosophic values of membership values. Finally we use the center of gravity method (Bai et al., 2006) to obtain a crisp value of :

$\operatorname{COG} C_{\eta}=\frac{\sum_{j=1}^{k} c_{j}\left(D_{\mu c}\right)}{\sum_{j=1}^{k}\left(D_{\mu c}\right)}$

The deneutrosophication of the other parameters are realized similar.

The obtained results from SNPV will be compared with Kahraman et al. (2015).

\section{Application}

\section{Present Value Analysis with Simplified Neutrosophic Sets}

In this section, we demonstrate the use of given formulas above. Table 1 shows data for investment alternative problem selection. Machinery Company planned to purchase a lathe among two models. For comparative purposes, the parameters are determined by the purchasing manager and two assistant purchasing managers. Assistant purchasing managers decided to make a compromise judgment since they have similar work experiences and knowledge. Hence, the experts' weights are 0.6 for the purchasing manager and 0.4 for assistant purchasing managers, respectively. The neutrosophic parameters of the alternatives are defined in Table 2 . 
Table 1

Neutrosophic Data for Investment Alternative Problem

\begin{tabular}{|l|c|}
\hline \multicolumn{2}{|c|}{ Lathe } \\
\hline Cost, dollar & $\{\langle c, T(c), I(c), F(c)\rangle \mid c \in C\}$ \\
\hline Annual Cost, dollar & $\{\langle a c, T(a c), I(a c), F(a c)\rangle \mid a c \in A C\}$ \\
\hline $\begin{array}{l}\text { Major Maintenance, dollar/ } \\
\text { 5th year }\end{array}$ & $\{\langle m m, T(m m), I(m m), F(m m)\rangle \mid m m \in M M\}$ \\
\hline Salvage Cost, dollar & $\{\langle s c, T(s c), I(s c), F(s c)\rangle \mid s c \in S C\}$ \\
\hline Interest Rate, year & $\{\langle l, T(l), I(l), F(l)\rangle \mid l \in I\}$ \\
\hline Useful Life, year & $\{\langle n, T(n), I(n), F(n)\rangle \mid n \in N\}$ \\
\hline
\end{tabular}

As an example, mathematical operations for alternative 2 were given. The evaluations of different experts are aggregated to obtain a single aggregated value by Equation 5. Table 3 represents the aggregated values of each parameter. For instance, the aggregated value of possible value of 35,000 is calculated as bellows;

$$
\begin{aligned}
& 1-\left((1-0.7)^{\left.0.6 *(1-0.5)^{0.4}\right)=0.632}\right. \\
& 1-\left((1-0.4)^{0.6} *(1-0.8)^{0.4}\right)=0.613 \\
& 1-\left((1-0.3)^{0.6} *(1-0.5)^{0.4}\right)=0.388
\end{aligned}
$$

Then, we calculated the deneutrosophicated value of each parameter. As show the process steps of formulas, an example of $\stackrel{C}{C}$ is given following;

$$
\begin{aligned}
& D_{C_{35,000}}=\frac{0.543+(1-0.462)}{2(1+0.765)} \\
& D_{C_{35,000}}=0.306
\end{aligned}
$$

The other possible values of $\stackrel{:}{C}$ are calculated.

$$
D_{C_{40,000}}=0.385 \quad D_{C_{45,000}}=0.220
$$

The next step is getting crisp values of $\stackrel{:}{C}$ by using Equation 23.

$$
\begin{aligned}
\text { COG } & \stackrel{:}{C}=\frac{(35,000 * 0.306)+(40,000 * 0.385)+(45,000 * 0.220)}{(0.127+0.240+0.154)} \\
& =39,524.07
\end{aligned}
$$

At the last step SNPV analysis formula (Equation 7) is applied

$$
\begin{aligned}
& S N P V=39,527.07+4,315.97\left(\frac{P}{A}, 29.734 \%, 9.862\right) \\
& +7,025.80\left(\frac{P}{F}, 29.734 \% .5\right)-6,579.92\left(\frac{P}{F}, 29.818 \%, 9.862\right) \\
& =\$ 42,116.04
\end{aligned}
$$

The same calculations are applied for Alternative 2 and calculation result is;

SNPV $=49,153.15+3,934.44\left(\frac{P}{A}, 29.818 \%, 9.734\right)+$

$6,902.528\left(\frac{P}{F}, 29.734 \%, 5\right)-6,369.355\left(\frac{P}{F}, 29.818 \%, 9.862\right)$ $=\$ 49,029.85$

Depending on SNPV analysis's results Alternative 1 is chosen as the best alternative

Table 3

\section{Neutrosophic Aggregation of Investment Parameters}

\begin{tabular}{ccc}
\hline Parameters & Possible Values & $\begin{array}{c}\text { Aggregated } \\
\text { Values }\end{array}$ \\
\hline$:$ & 35,000 & $\langle 0,632,0,613,0,388\rangle$ \\
$C$ & 40,000 & $\langle 0,592,0,690,0,428\rangle$ \\
$\ldots$ & 45,000 & $\langle 0,362,0,604,0,643\rangle$ \\
$:$ & 4,000 & $\langle 0,362,0,604,0,643\rangle$ \\
$A C$ & 4,500 & $\langle 0,745,0,327,0,262\rangle$ \\
$\ldots$ & 5,000 & $\langle 0,226,0,294,0,807\rangle$ \\
$:$ & 6,500 & $\langle 0,388,0,613,0,32\rangle$ \\
$M M$ & 7,000 & $\langle 0,543,0,765,0,462\rangle$ \\
$\ldots$ & 7,500 & $\langle 0,868,0,141,0,141\rangle$ \\
$:$ & 5,500 & $\langle 0,700,0,400,0,300\rangle$ \\
$S V$ & 6,500 & $\langle 0,500,0,800,0,500\rangle$ \\
$\ldots$ & 7,500 & $\langle 0,400,0,700,0,600\rangle$ \\
$:$ & 9 & $\langle 0,400,0,700,0,600\rangle$ \\
$\ldots$ & 10 & $\langle 0,848,0,161,0,161\rangle$ \\
$\ldots$. & 11 & $\langle 0,100,0,100,0,900\rangle$ \\
$:$ & 25 & $\langle 0,300,0,400,0,700)$ \\
$\boldsymbol{n}$ & 30 & $\langle 0,807,0,294,0,226\rangle$ \\
$\cdots$ & 35 & $\langle 0,226,0,294,0,807\rangle$ \\
\hline
\end{tabular}


The Possible Values and Neutrosophic Membership Values

\begin{tabular}{|c|c|c|c|c|c|c|}
\hline Parameters & $\begin{array}{c}\text { Possible Values } \\
\text { for Lathe } 1\end{array}$ & $\begin{array}{c}\text { Expert 1 } \\
(0.6) \\
\end{array}$ & $\begin{array}{c}\text { Experts 2-3 } \\
(0.4) \\
\end{array}$ & $\begin{array}{c}\text { Possible Values } \\
\text { for Lathe } 2\end{array}$ & $\begin{array}{c}\text { Expert 1 } \\
(0.6) \\
\end{array}$ & $\begin{array}{c}\text { Experts 2-3 } \\
(0.4)\end{array}$ \\
\hline \multirow[t]{3}{*}{ : } & $\$ 35,000$ & $\langle 0.5,0.8,0.5\rangle$ & $\langle 0.6,0.7,0.4\rangle$ & $\$ 45,000$ & $\langle 0.7,0.4,0.3\rangle$ & $\langle 0.5,0.8,0.5\rangle$ \\
\hline & $\$ 40,000$ & $\langle 0.7,0.4,0.3\rangle$ & $\langle 0.5,0.8,0.5\rangle$ & $\$ 50,000$ & $\langle 0.5,0.8,0.5\rangle$ & $\langle 0.7,0.4,0.3\rangle$ \\
\hline & $\$ 45,000$ & $\langle 0.3,0.4,0.7\rangle$ & $\langle 0.4,0.7,0.6\rangle$ & $\$ 55,000$ & $\langle 0.4,0.7,0.6\rangle$ & $\langle 0.3,0.4,0.7\rangle$ \\
\hline \multirow{3}{*}{$\begin{array}{c}: \\
A C \\
\ldots\end{array}$} & $\$ 4,000$ & $\langle 0.9,0.1,0.1\rangle$ & $\langle 0.8,0.2,0.2\rangle$ & $\$ 3,000$ & $\langle 0.4,0.7,0.6\rangle$ & $\langle 0.3,0.4,0.7\rangle$ \\
\hline & $\$ 4,500$ & $\langle 0.7,0.4,0.3\rangle$ & $\langle 0.6,0.7,0.4\rangle$ & $\$ 4,000$ & $\langle 0.7,0.4,0.3\rangle$ & $\langle 0.8,0.2,0.2\rangle$ \\
\hline & $\$ 5,000$ & $\langle 0.3,0.4,0.7\rangle$ & $\langle 0.5,0.8,0.5\rangle$ & $\$ 5,000$ & $\langle 0.3,0.4,0.7\rangle$ & $\langle 0.1,0.1,0.9\rangle$ \\
\hline \multirow{3}{*}{$\begin{array}{c}: \\
M M \\
\ldots\end{array}$} & $\$ 6,500$ & $\langle 0.5,0.8,0.5\rangle$ & $\langle 0.4,0.7,0.6\rangle$ & $\$ 5,500$ & $\langle 0.3 .0 .4,0.7$ & $\langle 0.5,0.8,0.5\rangle$ \\
\hline & $\$ 7,000$ & $\langle 0.8,0.2,0.2\rangle$ & $\langle 0.7,0.4,0.3\rangle$ & $\$ 6,500$ & $\langle 0.5,0.8,0.5\rangle$ & $\langle 0.6,0.7,0.4\rangle$ \\
\hline & $\$ 7,500$ & $\langle 0.6,0.7,0.4\rangle$ & $\langle 0.5,0.8,0.5\rangle$ & $\$ 7,500$ & $\langle 0.9,0.1,0.1\rangle$ & $\langle 0.8,0.2,0.2\rangle$ \\
\hline \multirow{3}{*}{$\begin{array}{c}: \\
S V \\
\ldots\end{array}$} & $\$ 5,500$ & $\langle 0.6,0.7,0.4\rangle$ & $\langle 0.5,0.8,0.5\rangle$ & $\$ 6,000$ & $\langle 0.7,0.4,0.3\rangle$ & $\langle 0.7,0.4,0.3\rangle$ \\
\hline & $\$ 6,500$ & $\langle 0.5,0.8,0.5\rangle$ & $\langle 0.4,0.7,0.6\rangle$ & $\$ 6,500$ & $\langle 0.5,0.8,0.5\rangle$ & $\langle 0.5,0.8,0.5\rangle$ \\
\hline & $\$ 7,500$ & $\langle 0.4,0.7,0.6\rangle$ & $\langle 0.7,0.4,0.3\rangle$ & $\$ 7,000$ & $\langle 0.4,0.7,0.6\rangle$ & $\langle 0.4,0.7,0.6\rangle$ \\
\hline : & $9 \%$ & $\langle 0.4,0.7,0.6\rangle$ & $\langle 0.4,0.7,0.6\rangle$ & $9 \%$ & $\langle 0.4,0.7,0.6\rangle$ & $\langle 0.4,0.7,0.6\rangle$ \\
\hline \multirow[t]{2}{*}{$\begin{array}{l}l \\
\ldots\end{array}$} & $10 \%$ & $\langle 0.8,0.2,0.2\rangle$ & $\langle 0.9,0.1,0.1\rangle$ & $10 \%$ & $\langle 0.8,0.2,0.2\rangle$ & $\langle 0.9,0.1,0.1\rangle$ \\
\hline & $11 \%$ & $\langle 0.1,0.1,0.9\rangle$ & $\langle 0.1,0.1,0.9\rangle$ & $11 \%$ & $\langle 0.1,0.1,0.9\rangle$ & $\langle 0.1,0.1,0.9\rangle$ \\
\hline : & 25 years & $\langle 0.3,0.4,0.7\rangle$ & $\langle 0.3,0.4,0.7\rangle$ & 25 years & $\langle 0.3,0.4,0.7\rangle$ & $\langle 0.3,0.4,0.7\rangle$ \\
\hline \multirow[t]{2}{*}{$\begin{array}{l}n \\
\cdots\end{array}$} & 30 years & $\langle 0.7,0.4,0.3\rangle$ & $\langle 0.9,0.1,0.1\rangle$ & 30 years & $\langle 0.7,0.4,0.3\rangle$ & $\langle 0.9,0.1,0.1\rangle$ \\
\hline & 35 years & $\langle 0.3,0.4,0.7\rangle$ & $\langle 0.1,0.1,0.9\rangle$ & 35 years & $\langle 0.3,0.4,0.7\rangle$ & $\langle 0.1,0.1,0.9\rangle$ \\
\hline
\end{tabular}

\section{Comparison with Classical Present Value Analysis}

In this section, we compare SNPV analysis and classical present value analysis. Classical present value analysis formula is given below. In order to get crisp data, we got the average of the possible values used in Table 3.

$$
P V=C+A C\left(\frac{P}{A}, l, n\right)+M M\left(\frac{P}{F}, l, t\right)-S V\left(\frac{P}{F}, l, n\right)
$$

Table 4 shows crisp data for investment alternative problem.

Table 4

Crisp Data for Investment Alternative Problem

\begin{tabular}{lcc}
\hline & Lathe 1 & Lathe 2 \\
\hline Cost, dollar & 40,000 & 50,000 \\
Annual Cost, dollar & 4,500 & 4,000 \\
Maintain Cost, dollar & 7,000 & 6,500 \\
Salvage Cost, dollar & 6,500 & 6,500 \\
Interest Rate, year & 10 & 10 \\
Useful Life, year & 30 & 30 \\
\hline
\end{tabular}

$$
\begin{aligned}
& P V_{1}=40,000+4,000\left(\frac{P}{A}, 30 \%, 10\right)+7,000\left(\frac{P}{F}, 30 \%, 5\right) \\
& -5,500\left(\frac{P}{F}, 30 \%, 10\right) \\
& P V_{1}=\$ 42,527.00 \\
& P V_{2}=45,000+3,000\left(\frac{P}{A}, 30 \%, 10\right)+7,500\left(\frac{P}{F}, 30 \%, 5\right) \\
& -6,000\left(\frac{P}{F}, 30 \%, 10\right)
\end{aligned}
$$

$$
P V_{2}=\$ 52,392.00
$$

Alternative 1 is selected according to the results of classical present value analysis. The ranking result of alternatives is the same as the SPVN method. 


\section{Comparison with Intuitionistic Present Value Analysis}

In this section, we compare our proposed SNPV analysis with the intuitionistic present value analysis in the literature. The intuitionistic parameters of the alternatives are defined in Table 5.

First, evaluations of many experts are aggregated to a single value. We used the intuitionistic fuzzy weighted averaging operator existing in the literature for comparative purpose. As an example, the parameter of $C_{C_{35,000}}$ is calculated as follows;

$1-\left((1-0,4)^{0,6}(1-0.6)^{0.4}\right)=0.489$

$(0.3)^{0.6} *(0.3)^{0.4}=0.300$

A single aggregated value of ${\stackrel{:}{C_{35,000}}}_{\text {in }}[0.489,0.300]$

Then, we calculate defuzzified values of intuitionistic membership values. A single aggregated value of $\dot{C}_{I}$ is calculated as follows;

$$
=0.489+0.5 *(1-0.300-0.489)
$$$$
=0.594
$$

Then, we calculate defuzzified values of each parameter.

$$
C_{I}=\frac{\left(\left(35,000 *(0.594)^{2}\right)+\left(4,000 *(0.670)^{2}\right)+\left(4,500 *(0.364)^{2}\right)\right)}{(0.594)^{2}+(0.670)^{2}+(0.364)^{2}}
$$

$$
C_{I}=38,819.30
$$

And finally, we find present value ;

$$
\begin{aligned}
& : \\
& P V=38,819.3+4,298.17\left(\frac{P}{A}, 29.766 \%, 9.833\right)+ \\
& 7.090,957\left(\frac{P}{F}, 29.766 \%, 5\right)-6.636,442\left(\frac{P}{F}, 29.766 \%, 9.833\right) \\
& : \\
& P V_{1}=\$ 41,415.22 \text { and } P V_{2}=\$ 50,895.71
\end{aligned}
$$

The Possible Values and Intuitionistic Membership Valu

\begin{tabular}{ccccccc}
\hline Parameters & $\begin{array}{c}\text { Possible Values } \\
\text { for Lathe 1 }\end{array}$ & $\begin{array}{c}\text { Expert 1 } \\
(\mathbf{0 . 6})\end{array}$ & $\begin{array}{c}\text { Experts 2-3 } \\
(\mathbf{0 . 4})\end{array}$ & $\begin{array}{c}\text { Possible Values } \\
\text { for Lathe 2 }\end{array}$ & $\begin{array}{c}\text { Expert 1 } \\
(\mathbf{0 . 6})\end{array}$ & $\begin{array}{c}\text { Experts 2-3 } \\
(\mathbf{0 . 4})\end{array}$ \\
\hline$:$ & $\$ 35,000$ & {$[0.4,0.3]$} & {$[0.6,0.3]$} & $\$ 45,000$ & {$[0.7,0.2]$} & {$[0.6,0.4]$} \\
$C_{I}$ & $\$ 40,000$ & {$[0.7,0.2]$} & {$[0.4,0.4]$} & $\$ 50,000$ & {$[0.5,0.4]$} & {$[0.6,0.4]$} \\
& $\$ 45,000$ & {$[0.2,0.6]$} & {$[0.4,0.5]$} & $\$ 55,000$ & {$[0.3,0.5]$} & {$[0.4,0.5]$} \\
$:$ & $\$ 4,000$ & {$[0.8,0.1]$} & {$[0.8,0.2]$} & $\$ 3,000$ & {$[0.3,0.6]$} & {$[0.4,0.5]$} \\
$A C_{I}$ & $\$ 4,500$ & {$[0.6,0.2]$} & {$[0.6,0.3]$} & $\$ 4,000$ & {$[0.6,0.2]$} & {$[0.6,0.3]$} \\
& $\$ 5,000$ & {$[0.3,0.5]$} & {$[0.4,0.6]$} & $\$ 5,000$ & {$[0.3,0.5]$} & {$[0.2,0.6]$} \\
$\vdots$ & $\$ 6,500$ & {$[0.4,0.6]$} & {$[0.3,0.5]$} & $\$ 5,500$ & {$[0.4,0.6]$} & {$[0.4,0.5]$} \\
$M M_{I}$ & $\$ 7,000$ & {$[0.7,0.1]$} & {$[0.7,0.1]$} & $\$ 6,500$ & {$[0.4,0.5]$} & {$[0.6,0.5]$} \\
& $\$ 7,500$ & {$[0.6,0.3]$} & {$[0.5,0.4]$} & $\$ 7,500$ & {$[0.8,0.2]$} & {$[0.7,0.2]$} \\
$:$ & $\$ 5,500$ & {$[0.5,0.3]$} & {$[0.4,0.6]$} & $\$ 6,000$ & {$[0.5,0.3]$} & {$[0.6,0.3]$} \\
$S V_{I}$ & $\$ 6,500$ & {$[0.4,0.3]$} & {$[0,4,0.5]$} & $\$ 6,500$ & {$[0.4,0.3]$} & {$[0.4,0.5]$} \\
& $\$ 7,500$ & {$[0.4,0.3]$} & {$[0.7,0.2]$} & $\$ 7,000$ & {$[0.4,0.5]$} & {$[0.4,0.4]$} \\
$:$ & $9 \%$ & {$[0.4,0.5]$} & {$[0.4,0.6]$} & $9 \%$ & {$[0.4,0.4]$} & {$[0.4,0.5]$} \\
$i_{I}$ & $10 \%$ & {$[0.7,0.1]$} & {$[0.8,0.2]$} & $10 \%$ & {$[0.7,0.2]$} & {$[0.8,0.2]$} \\
& $11 \%$ & {$[0.2,0.8]$} & {$[0.2,0.8]$} & $11 \%$ & {$[0.2,0.8]$} & {$[0.2,0.8]$} \\
& 25 years & {$[0.4,0.5]$} & {$[0.4,0.5]$} & 25 years & {$[0.3,0.5]$} & {$[0.4,0.6]$} \\
$:$ & 30 years & {$[0.7,0.2]$} & {$[0.7,0.1]$} & 30 years & {$[0.7,0.2]$} & {$[0.7,0.1]$} \\
$n_{I}$ & 35 years & {$[0.4,0.5]$} & {$[0.2,0.7]$} & 35 years & {$[0.3,0.5]$} & {$[0.2,0.7]$} \\
\hline
\end{tabular}


Alternative 1 is selected according to the results of intuitionistic present value analysis.

As it is seen from the calculations, the present values of each approach are different from the other approaches. This is because each approach has its own theoretical infrastructure. The obtained numerical results are summarized in Table 6. Since each approach requires different data inputs, obviously, it is not expected the same numerical results to be obtained. Each result has a specific meaning even the selected alternative is same. For instance, neutrosophic PVA has given the minimum present value for Alternative 2 with respect to others since indeterminacy parameter is considered in NSPV.

\section{Comparison of Methodologies}

Table 6

\begin{tabular}{|l|l|l|}
\hline & Alternative 1 & Alternative 2 \\
\hline $\begin{array}{l}\text { Present Value Analysis } \\
\text { with Simplified } \\
\text { Neutrosophic Sets }\end{array}$ & $\$ 42,116.04$ & $\$ 49,029.85$ \\
\hline $\begin{array}{l}\text { Comparison with Classical } \\
\text { Present Value Analysis }\end{array}$ & $\$ 42,527.00$ & $\$ 52,392.00$ \\
\hline $\begin{array}{l}\text { Comparison with } \\
\text { Intuitionistic Present } \\
\text { Value Analysis }\end{array}$ & $\$ 41,415.22$ & $\$ 50,895.71$ \\
\hline
\end{tabular}

\section{Comparison the Results of Arithmetic Average Aggregation Operator and Weighted Geometric Average Aggregation Operator}

In this section, we analyze the using of aggregation operators (Equation 5 and Equation 6) if there is a difference between the results. Ye (2014) put forward the weighted arithmetic average operator emphasizes group's major points, and then weighted geometric average operator emphasizes personal major points.

Present values by different aggregation operators are shown in Table 7.

Table 7

Comparison of Aggregation Operators

\begin{tabular}{|l|l|l|}
\hline & Alternative 1 & Alternative 2 \\
\hline $\begin{array}{l}\text { Weighted arithmetic } \\
\text { average operator }\end{array}$ & $\$ 42,116.04$ & $\$ 49,029.85$ \\
\hline $\begin{array}{l}\text { Weighted geometric } \\
\text { average operator }\end{array}$ & $\$ 42,912.34$ & $\$ 48,939.36$ \\
\hline
\end{tabular}

There is not too much change in the results according to the calculated results in Table 7 . Present value of Alternative 1 increases 72,88 dollars, and present value of Alternative 2 decreases 121,09 dollars. So, these results are closer to evaluation of the first expert.

The results of two aggregation operators can be analyzed more effectively if possible values of the firms are very close to each other.

\section{Conclusions}

Every company has investments in many forms which might be complimentary investments, prerequisite investments, substitute investments, and mutually exclusive investments. Costs and benefits from investments are one of the primary responsibilities of a finance manager to raise the company's profits.

Evaluation of investment alternatives is a decisionmaking problem under uncertainty. Therefore, decision maker's knowledge concerning interest rates, annual costs etc. are consist of vagueness and impreciseness. Fuzzy sets methodologies can handle the uncertainty of such problems.

Neutrosophic sets as a new extension of ordinary fuzzy sets bring forward a new point of view to the definition of membership functions. In this paper, present value analysis with simplified neutrosophic sets has been developed for the evaluation of investment alternatives. The proposed methodology helps decision makers to better express their knowledge, assessments and judgments on investment decision making problems. The proposed methodology uses the neutrosophic membership functions defined by decision makers. The proposed deneutrosophication equation transforms a neutrosophic set to a crisp set. Based on the deneutrosophicated values, the center of gravity methodology is used to get each parameter's crisp value. Thus, classical present value analysis formulas could be applied. Classical present value analysis and intuitionistic present value analysis have been compared. It has been observed that both methods suggest the same alternative.

The greatest feature that distinguishes this article from other studies is the first use of neutrosophic sets in investment analysis.

For future work, other investment analysis techniques such as neutrosophic annual value analysis, neutrosophic future value analysis, neutrosophic benefit/cost ratio analysis, neutrosophic rate of return analysis and payback period analysis can be developed for investment analysis problems.

\section{References}

Atanassov, K. T. (1986). Intuitionistic Fuzzy Sets. Fuzzy Sets and Systems, 20, 87-96. https://doi.org/10.1016/S01650114(86)80034-3

Bai, Y., \& Wang, D. (2006) Fundamentals of Fuzzy Logic Control — Fuzzy Sets, Fuzzy Rules and Defuzzifications. In: Bai Y., Zhuang H., Wang D. (eds) Advanced Fuzzy Logic Technologies in Industrial Applications. Advances in Industrial Control. Springer, London. https://doi.org/10.1007/978-1-84628-469-4

Bhattacharyya, R., Kumar, P., \& Kar, S. (2011). Fuzzy R\&D Portfolio Selection of Interdependent Projects. Computers \& Mathematics with Applications, 62(10), 3857-3870. https://doi.org/10.1016/j.camwa.2011.09.036 
Broumi, S., \& F. Smarandache, (2014). Single Valued Neutrosophic Trapezoid Linguistic Aggregation Operators Based Multi-Attribute Decision Making. Bulletin of Pure and Applied Sciences, 33, 135-155. https://doi.org/10.5958/23203226.2014.00006.X

Buckley, J. J. (1987). The Fuzzy Mathematics of Finance, Fuzzy Sets and Systems, 21, 257-273. https://doi: 10.1016/0165-0114(87) 90128-X

Chan, F. T. S., Chan, M. H., \& Tang, N. K. H. (2000). Evaluation Methodologies for Technology Selection. Journal of Materials Processing Technology, 107,330-337. https://doi.org/10.1016/S0924-0136(00)00679-8

Chiu, C. Y., \& Park, C. S. (1994). Fuzzy Cash Flow Analysis Using Present Worth Criterion. The Engineering Economist, 39(2), 113-138. http://dx.doi.org/10.1080/00137919408903117

David, G., Carmichael, A. Hersh M., \& Parasu P. (2011). Real Options Estimate Using Probabilistic Present Worth Analysis. The Engineering Economist, 56, 295-320. http://dx.doi.org/10.1080/0013791X.2011.624259

Demircan, M. L. (2016). Interval Type-2 Fuzzy Replacement Analysis in Call Center Investment Replacement Decision. International Journal of Management Replacement Replacement Decision, 130-136, 2 (10). http://www.iraj.in/journal/journal_file/journal_pdf/14-309-1480146330130-136.pdf

Dimitrovski, A., \& Matos, M. (2008). Fuzzy Present Worth Analysis with Correlated and Uncorrelated Cash Flows. C. Kahraman (Ed.). Fuzzy Engineering Economics with Applications. Springer-Verlag, Berlin Heidelberg. https://doi.org/10.1007/978-3-540-70810-0_2

Ejegwa, P. A. (2014). Correspondence between Fuzzy Multisets and Sequences. Global Journal of Science Frontier Research: Mathematics and Decision Sciences 14-7. 61-66. https://globaljournals.org/GJSFR_Volume14/5Correspondence-between-Fuzzy.pdf

Jahn, K. U. (1975). Intervall-wertige Mengen, Math. Nach, 68, 115-132. https://doi.org/10.1002/mana.19750680109

Kahraman, C., (Ed.). (2008). Fuzzy Engineering Economics with Applications, Studies in Fuzziness and Soft Computing, 233, Springer-Verlag, Berlin Heidelberg. https://doi.org/10.1007/978-3-540-70810-0.

Kahraman, C., Oztaysi B., \& Cevik, S. (2016). A Comprehensive Literature Review of 50 Years of Fuzzy Set Theory. International Journal of Computational Intelligence Systems, 3-24. https://doi.org/10.1080/18756891.2016.1180817

Kahraman C., Onar, C. S., \& Oztaysi B. (2015). Engineering Economic Analyses Using Intuitionistic and Hesitant Fuzzy Sets. Journal of Intelligent \& Fuzzy Systems 29, 1151-1168. https://doi.org/10.3233/IFS-151722

Kahraman, C., Ruan, D., \& Tolga, E. (2002). Capital Budgeting Techniques Using Discounted Fuzzy Versus Probabilistic Cash Flows. Information Sciences 142(1/4) 57-76. https://doi.org/10.1016/S0020-0255(02)00157-3

Kahraman C., Onar S. C., \& Oztaysi B. (2018) Present Worth Analysis Using Pythagorean Fuzzy Sets. In: Kacprzyk J., Szmidt E., Zadrożny S., Atanassov K., Krawczak M. (eds) Advances in Fuzzy Logic and Technology 2017. IWIFSGN 2017, EUSFLAT 2017. Advances in Intelligent Systems and Computing, vol 642. Springer, Cham. https://doi.org/10.1007/978-3-319-66824-6_30

Karsak, E. E., \& Tolga E. (2001). Fuzzy Multi-Criteria Decision Making Procedure for Evaluating Advanced Manufacturing Investments. International Journal of Production Economics 69, 49-64. https://doi.org/10.1016/S0925-5273(00)00081-5

Kaufmann, A., \& Gupta, M. M. (1988). Fuzzy Mathematical Models in Engineering and Management Science. Amsterdam: Elsevier, 19-35, 159-165.

Kharal, A. (2014). A Neutrosophic Multi-Criteria Decision Making Method, New Mathematics and Natural Computation, 10, 143-162. http://www.worldscientific.com/doi/abs/10.1142/S1793005714500070

Kuchta D. (2001). A Fuzzy Model For R\&D Project Selection With Benefit, Outcome and Resource Interactions. The Engineering Economist, 46(3), 164-180. http://dx.doi.org/10.1080/00137910108967571

Kuchta, D. (2008). Fuzzy Rate of Return Analysis and Applications in Fuzzy Engineering Economics with Applications, C. Kahraman Ed., Berlin: Springer-Verlag. http://www.springer.com/us/book/9783540708094

Nicolas, W. (2015). Fuzzy Classification of Online Customers, 7-26. Switzerland: Springer. http://www.springer.com/cn/book/9783319159690

Omitaomu, O. A., \& Badiru, A. (2007). Fuzzy Present Value Analysis Model for Evaluating Information System Projects. The Engineering Economist, 52(2), 157-178. http://dx.doi.org/10.1080/00137910701328912

Sambuc, R. (1975). Fonctions $\phi$-floues. Application l'aide au diagnostic en pathologie thyroidienne, in, Thesis Univ. Marseille, France. 
Smarandache, F. A. (1998). Unifying Field in Logics. Neutrosophy: Neutrosophic Probability, Set and Logic. Rehoboth: American Research Press. https://arxiv.org/ftp/math/papers/0101/0101228.pdf

Yager, R. R. (1986). On the theory of bags, International Journal of General Systems, 13, $23-37$. http://dx.doi.org/10.1080/03081078608934952

Sheen, J. N. (2005). Fuzzy Financial Profitability Analyses of Demand Side Management Alternatives from Participant Perspective. Information Sciences, 169(3/4), 329-364. https://doi.org/10.1016/j.ins.2004.05.007

Torra, V. (2010). Hesitant Fuzzy Sets. International Journal of Intelligent Systems, 25, 529-539. http://dx.doi.org/10.1002/int.20418

Ward, T. L. (1985). Discounted Fuzzy Cash Flow Analysis. Proceedings of 1985 Fall Industrial Engineering Conference, Institute of Industrial Engineers, 476-481.

Wen, I., \& Cheng H. D. (2013). A Novel Neutrosophic Logic Svm (N-Svm) and Its Application to Image Categorization. New Mathematics and Natural Computation, $27-42$. http://www.worldscientific.com/doi/pdf/10.1142/S1793005713500038

Xu, B., Fang, W., Shi, R., Yu, J., \& Liu, L. (2009). Three-Objective Fuzzy Chance-Constrained Programming Model for Multiproject and Multi-Item Investment Combination. Information Sciences, 179(5), 623-641. https://doi.org/10.1016/j.ins.2008.10.031

Xu, J., Zheng, H., Zeng, Z., Wu, S., \& Shen, M. (2012). Discrete Time Cost Environment Trade-Off Problem for LargeScale Construction Systems with Multiple Modes Under Fuzzy, Uncertainty and Its Application to Jinping-Ii Hydroelectric Project. International Journal of Project Management, 30, 950-966. https://doi.org/10.1016/j.ijproman.2012.01.019

Xu, Z. S. (2012). Intuitionistic Fuzzy Multi-Attribute Decision Making: An Interactive Method. IEEE Transactions on Fuzzy Systems, 20,514-525. http://ieexplore.iee.. org/stamp/stamp.jsp?arnumber=6087279 https://doi.org/10.1109/TFUZZ.2011.2177466

Ye, J. A. (2014). Multicriteria Decision-Making Method Using Aggregation Operators for Simplified Neutrosophic Sets. Journal of Intelligent \& Fuzzy Systems, 26, 2459-2466. https://doi.org/10.3233/IFS-130916

Zadeh, L. A. (1965). Fuzzy Sets. Information and Control, 8, 338-353. https://doi.org/10.1016/S0019-9958(65)90241-X

Zadeh, L. A. (1975). The Concept of a Linguistic Variable and Its Application to Approximate Reasoning-1. Information Sciences, 8, 199-249. http://citeseerx.ist.psu.edu/viewdoc/download?doi=10.1.1.335.1122\&rep=rep1\&type=pdf https://doi.org/10.1016/0020-0255(75)90036-5

The article has been reviewed.

Received in October, 2017; accepted in June, 2018. 